\title{
IMPACT OF TYPHOON VONGFONG 2014 ON THE IONOSPHERE AND GEOMAGNETIC FIELD ACCORDING TO SWARM SATELLITE DATA: 1. WAVE DISTURBANCES OF IONOSPHERIC PLASMA
}

\author{
V.I. Zakharov \\ Lomonosov Moscow State University, \\ Moscow, Russia,zvi_555@list.ru \\ A.M. Obukhov Institute of Atmospheric Physics RAS, \\ Moscow, Russia,_zvi_555@list.ru

\section{V.A. Pilipenko} \\ Schmidt Institute of Physics of the Earth RAS, \\ Moscow, Russia, pilipenko_va@mail.ru \\ Space Research Institute RAS, \\ Moscow,Russia, space.soliton@gmail.com
}

\author{
V.A. Grushin \\ Space Research Institute RAS, \\ Moscow, Russia,vgrushin@iki.rssi.ru

\section{A.F. Khamidullin} \\ Lomonosov Moscow State University, \\ Moscow, Russia, askarhamidullin@gmail.com
}

\begin{abstract}
The article considers the influence of large atmospheric processes on the ionosphere by the example of tropical typhoon Vongfong 2014. We use data obtained from three SWARM satellite missions (450-500 km altitude). We discuss two possible mechanisms of transfer of atmospheric disturbances to ionospheric heights. The first mechanism is the generation of acoustic-gravity waves (AGWs); the second mechanism considers the excitation of electric fields in the atmosphere. We propose new techniques for detecting the ionospheric response to AGW, which rely on loworbit satellite data. The first technique is based on determination of relative electron density variations in the
\end{abstract}

frequency band from 15 to $150-180 \mathrm{~s}$, corresponding to certain scales of AGW. The second technique estimates space-time derivatives of the electron density, measured by two nearby SWARM satellites. We present and estimate the characteristic magnitudes of ionospheric response effects, their localization and spatial-temporal characteristics for the large tropical cyclone under study.

Keywords: tropical cyclone, ionosphere, electron density, acoustic-gravity wave, SWARM, equatorial anomaly.

\section{IONOSPHERIC PLASMA DISTURBANCES CAUSED BY TYPHOONS}

Earth's upper atmosphere and ionosphere are affected by various processes, which occur both in near-Earth space and in the lower atmosphere. It is clear that mechanisms of transfer of atmospheric effects to ionospheric heights and physics of ionospheric responses to atmospheric phenomena are underexplored. They represent not a consistent theory, but a set of several assumptions that are physically justified and in some cases experimentally verified. The atmosphere-ionosphere coupling can be considered by the example of geophysical processes with large energy release - tropical cyclones (TCs) that affect several geophysical shells. TC is a powerful atmospheric vortex. At wind speeds greater than $\sim 30 \mathrm{~m} / \mathrm{s}$, TC becomes a typhoon. Each year on Earth at latitudes between $5^{\circ}$ and $20^{\circ}$ in both hemispheres, $\sim 100$ TCs occur. TC is an effective mechanism for releasing the excess heat in the ocean-atmosphere system when convection and global circulation become insufficient. Inside each TC there is a region $\sim 30 \mathrm{~km}$ in diameter in which the wind speed is close to zero - the eye of the cyclone. At a distance of a few hundreds of kilometers from the center of the eye, the wind speed reaches a maximum and drops to the TC periphery. In height, TC occupies almost the entire troposphere $(\sim 20 \mathrm{~km})$.

Powerful meteorological disturbances in the atmosphere can affect Earth's ionosphere, but the mechanism of transfer of disturbances from powerful atmospheric phenomena (thunderstorm activity, tropical cyclones, hurricanes, tornadoes) to the ionosphere is currently unknown. As possible mechanisms several processes are considered which can explain the observed phenomena more or less satisfactorily. The ionospheric response to dynamic processes in the lower atmosphere may be associated with acoustic gravity waves (AGWs) comprising internal gravity waves (IGWs) and acoustic waves, separated by the Brunt-Väisälä frequency $\Omega_{b}$ ( 6-10 min periods) [Danilov et al., 1987; Hocke, Schlegel, 1996]. AGWs are excited by powerful meteorological phenomena in the lower atmosphere and then propagate to ionospheric heights. Wave modulation of the ionospheric electron density $N_{\mathrm{e}}$ manifests itself in the generation of traveling ionospheric disturbances (TID), which are detected using radiophysical methods. According to the estimates made by Forbes et al. [2000], the $N_{\mathrm{e}}$ variability at the F-layer maximum, caused by the influence of meteorological factors, can be as high as $35 \%$ of the background level under quiet geomagnetic conditions. Calculated TID azimuths and horizontal velocities of meteorological origin suggest that probable zones of generation of the recorded TIDs are in the troposphere and coincide with low atmospheric pressure zones in cyclone formation regions [Bertin et al., 1975]. Statistical studies of 24 strong typhoons of 1987-1992 have shown that the medium-scale TIDs are often identified which are caused by lower atmosphere turbulization and AGW emission, when a strong typhoon comes ashore or is near the coast [Xiao et al., 2007]. 
Wave transfer is perhaps the most effective mechanism of energy extraction from the atmospheric turbulence generation region. The wave mechanism is primarily associated with IGWs and acoustic waves, which have different frequencies and dispersion laws. In the frequency range of the acoustic branch of AGWs $(<10$ min periods) there are also magnetospheric magnetohydrodynamic (MHD) waves (Pc5 geomagnetic pulsations) generated by Alfvén oscillations, which can modulate the ionospheric plasma density. However, neither AGW frequencies nor MHD-wave frequencies on a LEO satellite moving at a velocity $V_{c}$ exceeding the wave disturbance velocity can be measured, and the observed frequency of the disturbance $f$ is determined by spatial scales of the turbulence region in the lower atmosphere $L: f \sim V_{\mathrm{c}} / L$.

In the region of intense cyclonic activity, external current can also emerge whose density is several orders of magnitude greater than the background one [Isaev et al., 2002]. As a result, throughout the ionosphere over TC the quasi-stationary electric field may increase by $1-2$ orders of magnitude. The channel of influence of atmospheric processes on ionospheric plasma disturbances may also be associated with the formation of an additional electric field generated by movements of the neutral component in the lower ionosphere [Pokhotelov et al., 1994]. Entrainment of charged particles by ionospheric neutral component flows leads to the local generation of electric currents and plasma density perturbations. The excited current system comprises field-aligned currents, which transfer a disturbance to the upper ionosphere. The observed increase in $N_{\mathrm{e}}$ in the F2 layer during Hurricane Katrina was presumably caused by the electric field effect [Bondur et al., 2008]. The electric fields in the ionosphere generated during intense weather events can affect the trapped particles of the inner radiation belt at low heights [Melioransky, 2007].

In this paper, in view of the complexity of the problem and the lack of experimental data required to solve it, we do not attempt to classify the type of electron density modulation in the ionosphere, but from physical considerations we analyze the ionospheric response and determine its parameters.

The ionospheric response to typhoons was detected using radiophysical methods of ionosphere monitoring: observation of Doppler frequency shift of HF radio signals [Huang et al., 1985; Xiao et al., 2007], sounding of the lower ionosphere along VLF radio paths [Rozhnoi et al., 2014] and during rocket experiments [Vanina-Dart et al., 2007]. Techniques for remote diagnostics of the ionosphere by measuring temporal variations of the ionospheric total electron content (TEC) from GPS signals are most actively being developed [Hofmann-Wellenhof et al., 1992]. New opportunities offered by the technique for spatial GPS detector [Ho et al., 1996; Afraimovich, Perevalova, 2006] can be used to identify ionospheric disturbances of meteorological origin in a given region of Earth. The technique for the analysis of data from GPS-receiver networks has been successfully utilized to identify different ionospheric phenomena [Ho et al., 1996] associated with solar eclipses, explosions and earthquakes, geomagnetic storms, and solar flares [Afraimovich, Perevalova, 2006; Zakharov et al., 2008]. At the maximum of high-power cyclones there were $N_{\mathrm{e}}$ oscillations with periods characteristic of AGWs of different scales (from 2 to $60 \mathrm{~min}$ ) [Zakharov, Kunitsyn, 2012; Polyakova, Perevalova, 2013]. The average amplitude of large-scale (T 20-60 min) TEC oscillations is 2-3 times higher than the level of background fluctuations, and for small-scale ( $T<20 \mathrm{~min}$ ) variations the amplitude increases 1.5-2 times. The size of the region covered by disturbances can reach several thousands of kilometers, being usually larger in the ionosphere than in the atmosphere. The intensity of ionospheric plasma disturbances is proportional to the power of the cyclone [Polyakova, Perevalova, 2013].

Zakharov, Kunitsyn [2012], using statistically significant material, have shown that the GPS observation technique is quite effective in identifying the response of the upper atmosphere to global synoptic processes by correlation methods. It has been found that the wave structures detected by GPS interferometry in the selected observation network are geographically related to places of orographic disturbances. The authors observed that AGW can be generated at the coast flown around by air masses captured by the typhoon. Such wave structures are excited most efficiently at a high rate of development or destruction of the typhoon, with the wave structures most pronounced in areas where air masses move at a high velocity, i.e. their generation is not connected with the eye of the typhoon. Simultaneous consideration of GPS data and radio occultation sounding technique has found deep, $\sim 50 \%, N_{\mathrm{e}}$ modulation in the F2 layer in the storm area, with the ionospheric anomalies located on the periphery of the typhoon, not near its eye [Li et al., 2018]. However, the question about effectiveness of the use of the GPS techniques in studying the ionospheric response to weather events is still open. For example, Afraimovich et al. [2008] when analyzing integral TEC maps failed to identify an explicit response to a typhoon. Vanina-Dart et al. [2007] have revealed a decrease in $N_{\mathrm{e}}$ over a tropical cyclone at E-layer heights, but have not found effects of the cyclone at F2-layer heights.

Effects of atmospheric disturbances in the ionosphere are also searched using data from LEO satellites [Gokhberg et al., 1996]. Measurements on the COSMOS-1809 satellite (at $800 \mathrm{~km}$ ) have revealed localized disturbances of quasi-stationary electric field up to 25 $\mathrm{mV} / \mathrm{m}$ and concomitant $N_{\mathrm{e}}$ changes to $6 \%$ over regions with strong atmospheric disturbances [Sorokin et al., 2005; Isayev et al., 2010].

In general, the brief review of the relationships between atmospheric and ionospheric processes indicates considerable difficulties in recording and ambiguity in interpreting the observed phenomena. The common difficulties in identifying the influence of even major events on the ionosphere in the geosphere system are also associated with the fact that the ionosphere is an open system which is affected by near-Earth processes in the magnetosphere (magnetic storms and substorms) 
and solar events (flares, coronal mass ejections). In this regard, some researchers believe that the facts of detection of the F-layer response to TC are insufficiently statistically valid or simply unique [Rishbeth, 2006].

The purpose of this series of works is the experimental study of the effects of a powerful tropical cyclone on the ionosphere, the determination of the amplitude and spatial-temporal scale of these effects based on electromagnetic and plasma measurements made by SWARM satellites when they pass over typhoons. Due to heterogeneity and extensiveness of the material in use, this paper is divided into two independent parts united by a common subject of study. The first part deals with the results of the identification of wave-like structures in the ionospheric electron density, measured with Langmuir probes of the SWARM project. The second part examines typhoon-related geomagnetic disturbances recorded with onboard magnetometers.

\section{DATA AND THEIR ANALYSIS}

The SWARM project consists of three satellites (A, $\mathrm{B}$, and $\mathrm{C}$ ) in the two circumpolar orbits. Satellites A and $\mathrm{C}$ are close together at a height of $\sim 470 \mathrm{~km}$ in a similar orbital plane (inclination of $87.4^{\circ}$ ), spaced by $\sim 1.4^{\circ}$ in longitude with a $2-10 \mathrm{~s}$ difference in orbital periods. The orbit of satellite $\mathrm{B}$ is shifted relative to the orbits of satellites $\mathrm{A}$ and $\mathrm{C}$ by $\sim 135^{\circ}$, has an inclination of $88^{\circ}$, and is at a height of $\sim 510 \mathrm{~km}$ [Olsen et al., 2013 ]. Satellite velocities are $7-7.5 \mathrm{~km} / \mathrm{s}$. In this paper, we use $N_{\mathrm{e}}$ measurements made with Langmuir probes onboard satellites with a frequency of $2 \mathrm{~Hz}$. The minimum ion density recorded by Langmuir probes is at least $10^{2}$ ions $/ \mathrm{cm}^{3}$ [http://directory.eoportal.org/web/eoportal/satellitemissions/ s/swarm]. This allows us, in the assumption of plasma quasi-neutrality, to estimate the error in determining $N_{\mathrm{e}}$ in the F2 layer to be less than $1 \%$. The identified effects given below exceed the said error of the equipment.

The data on TC trajectory were taken from the database [http://weather.unisys.com/hurricane]. They contain the temporal dynamics of the cyclone and its geographical reference.

The study of TC effects, despite them being powerful and having a potential effect on the ionosphere, has one important feature that makes it difficult to unambiguously interpret the ionospheric effects. TCs occur at low latitudes and often geographically coincide with the equatorial anomaly (EA) in the ionosphere [Walker, 1981; Balan et al., 2018]. EA manifests itself as ionization crests at the F2-layer maximum on both sides of the equator at $15^{\circ}-20^{\circ}$ latitudes and depressions over the geomagnetic equator. A cause of EA is the fountaineffect caused by the plasma drift upward over the geomagnetic equator followed by its northward and southward spreading along magnetic field lines.

The idea for identifying the ionospheric response is related to the difference between spatiotemporal characteristics of the processes under study. The EA region occupies a range of near-equatorial latitudes of not less than $\sim 20^{\circ}$, while the TC effect should be localized at a smaller latitudinal region conventionally associated with the central TC region.
As noted above, in a LEO satellite the observed frequency of a disturbance is determined by spatial scales of waves $L$ due to the Doppler effect. In our approach, ionospheric disturbances are regarded as a stationary wave because the satellite velocity exceeds the AGW velocity by more than an order of magnitude. Spatial scales of the storm area in the atmosphere for TCs we analyze are less than $1500 \mathrm{~km}$. If we consider TC to be the source of ionospheric disturbances, then, taking into account SWARM satellite velocities, periods of ionospheric disturbances should be not longer than 150-200 $\mathrm{s}$. The high-frequency limit of the filter in this case may correspond to the spatial scales of the order of 30-50 $\mathrm{km}$. In line with these estimates, we have chosen bandpass filter parameters from $10-15$ to $150-180 \mathrm{~s}$.

Figure 1 shows the temporal dynamics of typhoon Vongfong of the highest category V on the SaffirSimpson hurricane wind scale [http://www.nhc.noaa.gov/aboutsshws.php]. The typhoon developed in the near-equatorial part of the Pacific Ocean from the tropical depression on October 2-14, 2014. The wind speed in the maximum phase on October 7-10 was almost $80 \mathrm{~m} / \mathrm{s}$. Geomagnetic and solar conditions during the typhoon were quiet or moderately disturbed $\left(K_{\mathrm{p}}<4\right.$, and $\left.|D s \mathrm{t}|<15 \mathrm{nT}\right)$. This fact allows us to slightly simplify the interpretation of the observed phenomena.

AGW generated by TC represents a quasi-spherical wave diverging at angles up to $40^{\circ}$ according to the dispersion relation for ionospheric heights. Depending on the position of the satellite trajectory relative to the disturbance, which can be regarded as a torus-like structure in a rough approximation, we can get: 1) two regions of successive spatiotemporal changes in $\mathrm{Ne}$ (increase, and then decrease), when a satellite passes near the projection of the wave disturbance source on the ionosphere; 2) one such region of $\mathrm{Ne}$ perturbation, if a satellite passes along the periphery of the disturbance region, i.e. far from the point of projection of the disturbance center on the ionosphere. In real conditions, the situation is much more complicated because the source of the disturbance is clearly extended, and the $\mathrm{Ne}$ perturbations result from a superposition of wave harmonics with different wave vectors. Thus, the observed effects depend not only on the configuration

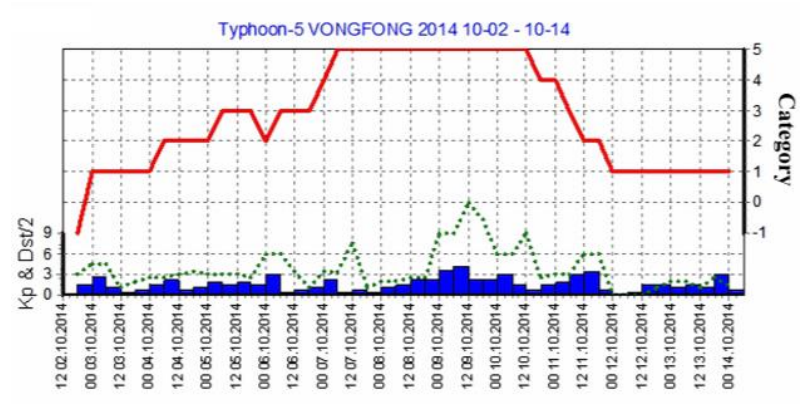

Figure 1. Temporal dynamics of typhoon Vongfong on October 2-14, 2014. The time evolution of the phenomenon on the Saffir-Simpson hurricane wind scale is shown along the right axis (the conditional value -1 corresponds to depression). The $K_{\mathrm{p}}$ (histogram) and Dst (dotted line) geomagnetic indices are plotted along the left axis 
of the wave disturbance, but also on how the probe passes through the disturbance region. From the trajectory calculations we determined moments of satellite passages over the TC region and then analyzed SWARM data related to this time range.

\section{RESULTS OF THE ANALYSIS}

Let us give an example of the cyclone effect against EA. Figure 2 shows projections of SWARM A and C tracks on Earth's surface on October 7, 2014 from 13:20 to $13: 35$ UTC.

Color of the tracks corresponds to the measured $N_{\mathrm{e}}$ disturbance. Intensity of the hurricane is marked with color according to the Saffir-Simpson hurricane wind scale: warmer color indicates a more powerful phase of the event (see Figure 1). The two satellites are seen to fly almost synchronously (with a difference of no more than $10 \mathrm{~s}$ ) into the storm area, conventionally depicted as a circle with a radius of $\sim 1000 \mathrm{~km}$ (ellipse in the projection of the Figure). The satellites entered this area at $\sim 13: 24$ UTC and left it at $\sim 13: 29$ UTC.

To identify the ionospheric response, a special procedure of bandpass polynomial filtering has been applied. When constructing filtering polynomials separately for high- and low-frequency components of the signal under study, their order was adaptively defined as a problem solution giving the minimum standard deviation from experimental data for the given window size within the selected error. The numerical experiment has demonstrated efficiency in identifying the wave-like disturbance against the model time variation in the electron density, which differs in period several times from the disturbance of interest and exceeds it in amplitude not less than 100 times. The possibility of constructing such a scheme of identification is associated with characteristic spatial scales of the structures. The chosen frequency band corresponds to spatial characteristics of the disturbance considered and depends on the satellite velocity.

The ionospheric response to the typhoon thus detected is shown in Figure 3, which depicts relative Ne variations in the frequency band 15-150 s. The correlation coefficient of these variations between satellites A and $\mathrm{C}$ is more than 0.92 , with its maximum corresponding to the time shift of $3 \mathrm{~s}$. During normal operation of the SWARM system, the difference between orbital periods of these satellites has a nominal value from 2 to $10 \mathrm{~s}$ [http://directory.eoportal.org/web/eoportal/satellite -missions/s/swarm]. The disturbance occurring at $\sim 13: 24$ UTC signifies the satellites' ingress into the storm area; and at 13:29 UTC, its egress from the zone of coverage of the typhoon. The observed effect with the relative amplitude $\sim 5-8 \%$ in the band under study exceeds the level of intrinsic measurement noise $\sim 1-2$ $\%$. The detected response is usually asymmetric.

Typhoon-5 VONGFONG vs SWARM Ne current $5 \mathrm{~min}$ 2014-10-07 13-20-13-35

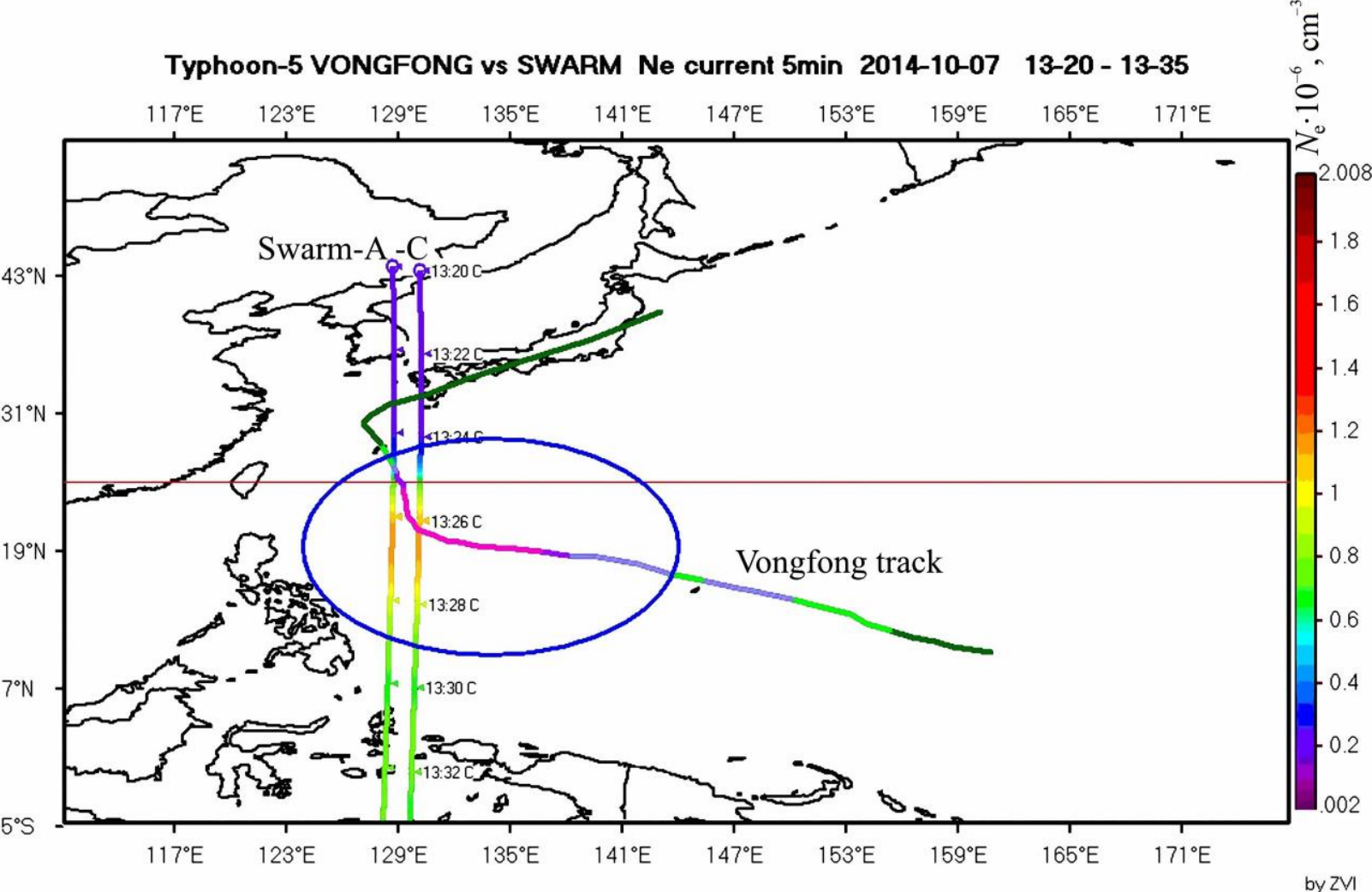

$5 \circ 5$

Figure 2. Geographical reference of projections of SWARM A and C tracks on Earth's surface on October 7, 2014. Close to the tracks are UTC moments with a step of $2 \mathrm{~min}$. The track of typhoon Vongfong throughout its lifetime is indicated by a broken line starting at a point with coordinates $\left(7.80^{\circ} \mathrm{N}, 160.50^{\circ} \mathrm{E}\right)$. Color of the satellite tracks corresponds to the electron density whose scale is given on the right panel. Intensity of the hurricane is marked with color according to the Saffir-Simpson wind scale, see Figure 1. 
07.10.2014, signal $15-150 \mathrm{~s}$

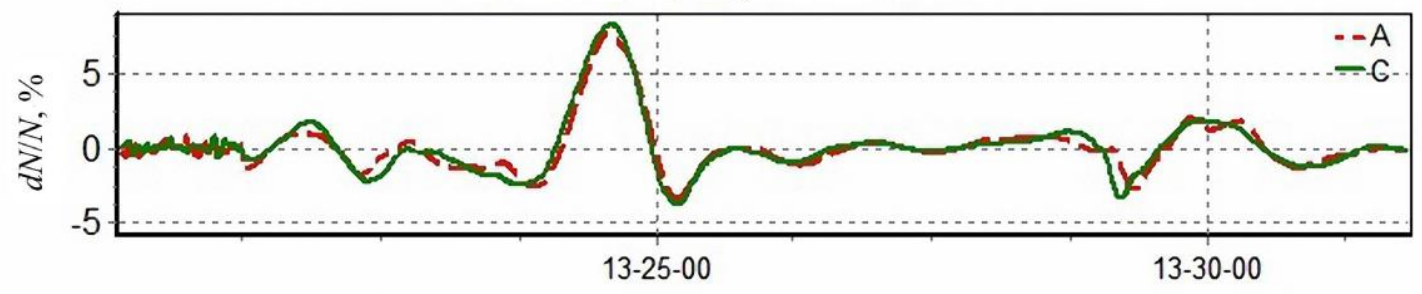

Figure 3. Relative ionospheric response to the typhoon in the electron density $N_{\mathrm{e}}$ in frequency band corresponding to 15-180 s wave structure periods

The response was identified against the measured $N_{\mathrm{e}}$ when the satellite passed over the equatorial anomaly approximately from 13:24 to 13:40 UTC (Figure 4). The contributions from the two processes were separated through filtration in a physically based frequency band.

Similar data during the typhoon considered have been acquired in more than ten cases both for the pair of satellites A, C, and for the single satellite B. For example, Figure 5 presents the result of detection of TC effect on ionospheric plasma at $~ 12: 52$ UTC (ingress) and 12:57 UTC (egress) on October 11, 2014.

All of the above allows us to conclude that the technique for analyzing disturbances in a selected frequency band corresponding to spatial scales of AGW may be used to identify TC effects on ionospheric plasma. A disturbance generated by a large cyclone can reach $10 \%$ of the current values of $N_{\mathrm{e}}$ in a specific frequency band, which is pronounced against regular variations. For additionally considered cyclones - Hagupit, Halong, 2014, we have also detected TC-induced $N_{\mathrm{e}}$ perturbations with relative amplitudes up to $10 \%$ exceeding recording errors. This result can be considered in general as a confirmation of the wave mechanism of transfer of disturbance to ionospheric heights of AGW with scales of the order of thousands of kilometers. The results indicate the spatial localization of disturbance on the periphery of TC, i.e. outside the eye of the cyclone, which is consistent with the conclusions drawn by Li et al. [2018].

SWARM A and C with identical equipment, closely spaced in latitude, constitute in fact a mobile "gradient installation" in the upper ionosphere. Due to data from this space gradientometer, we can propose another method of detecting TC-induced disturbances, which relies on the analysis of derivatives of recorded parameters. Precise information on satellites' trajectories allows us to evaluate not only temporal but also spatial gradients of the electron density with the accuracy sufficient to solve the problem.

Figures 6-8 show time (Figure 6), height (Figure 7), and longitude (Figure 8) derivatives of the measured $N_{\mathrm{e}}$.

The calculated derivatives exhibit the spatialtemporal structure of ionospheric disturbance depending on the configuration of the wave disturbance and on which region of this disturbance the satellites with onboard Langmuir probes pass over. Global maxima and minima of the derivatives in the Figures correspond to the time when the TC effect on ionospheric plasma might have been observed. The reasonings about shape of the expected response, which have been discussed in the previous section, are, of course, simplified. The spatial derivatives show in general a rather complicated internal structure of the dynamics of electron density perturbation, which results from the superposition of harmonics of waves of different origin.

Figure 6 illustrates the time evolution of the time derivative $d N_{\mathrm{e}} / d t$, which, strictly speaking, is the total derivative with respect to all three elements of the trajectory: height, latitude, and longitude. Despite some instability of the estimates, the Figure shows areas corresponding to TC-induced oscillations.

The presence of the spatially localized disturbance is convincingly confirmed when precision tracking of two nearby SWARM satellites is used. The spatial derivatives in height and longitude (see Figures 7, 8) confidently show a localized ionospheric disturbance associated in time with the cyclone. When the satellite passes over the disturbance, it is the longitude derivative that is directed to the center of the disturbance and hence is maximum. The sign of the derivatives $d N_{\mathrm{e}} / d h$ and $d N_{\mathrm{e}} / d l o n$ indicates that $N_{\mathrm{e}}$ decreases with height and satellites pass over the "slope" of the disturbance whose amplitude decreases in the direction from the eye of TC.

The measurements made in the experiment in principle provide sufficiently robust estimates of $N_{\mathrm{e}}$ and allow us to use them for assessing the TC effects on the ionosphere. The comparison between latitude-longitude derivatives shows that the longitude derivatives, i.e. in the direction perpendicular to the track, are most informative in terms of the identification of the ionospheric response to atmospheric cyclones. The general analysis of data on various cyclones indicates that the response in longitude is about 100 times larger than that in latitude.

\section{DISCUSSION AND CONCLUSIONS}

The ionospheric wave response to major events in the atmosphere manifests itself in the generation of acoustic oscillations by atmospheric turbulence [Gossard, Hooke, 1975], which propagate from ionospheric heights and modulate the electron density. During the maximum development of powerful TCs there are electron density fluctuations with scales characteristic of large-scale AGWs. The size of the region covered by disturbances can reach several thousands of kilometers, being usually larger in the ionosphere than in the atmosphere.

The theoretical analysis shows that the intensity of AGW generated by atmospheric turbulence sharply 


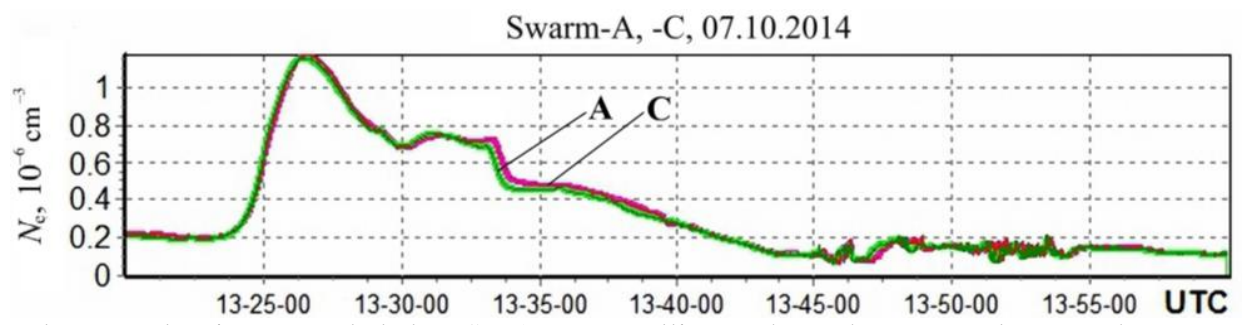

Figure 4. Electron density recorded by SWARM satellites when they passed over the equatorial anomaly

Swarm, 07.10.2014

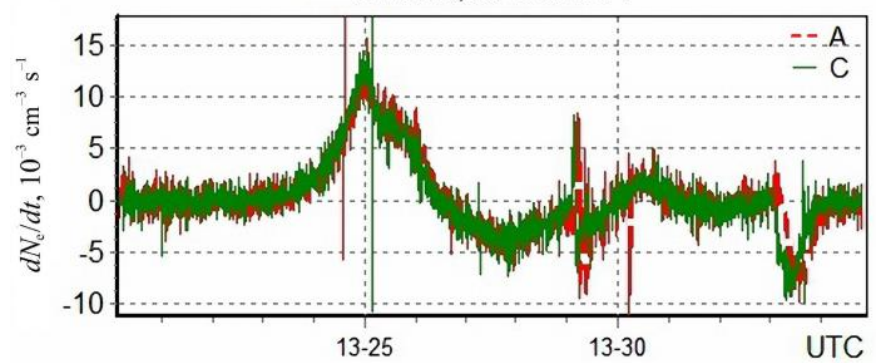

Figure 5. Result of identification of the wave structure related to TC effects on the ionosphere using data from SWARM A and $\mathrm{C}$ probes in the period band $15-180 \mathrm{~s}$

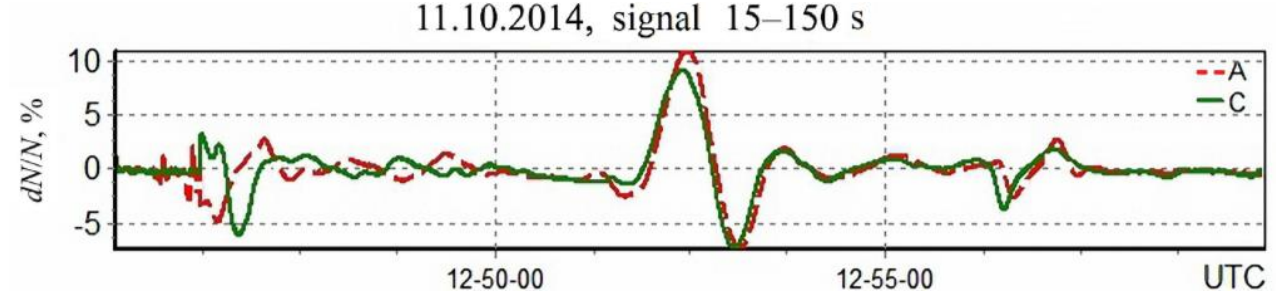

Figure 6. Identification of cyclone effects using the time derivative of electron density by SWARM A and C, on October 7, 2014, 13:20-13:35 UTC

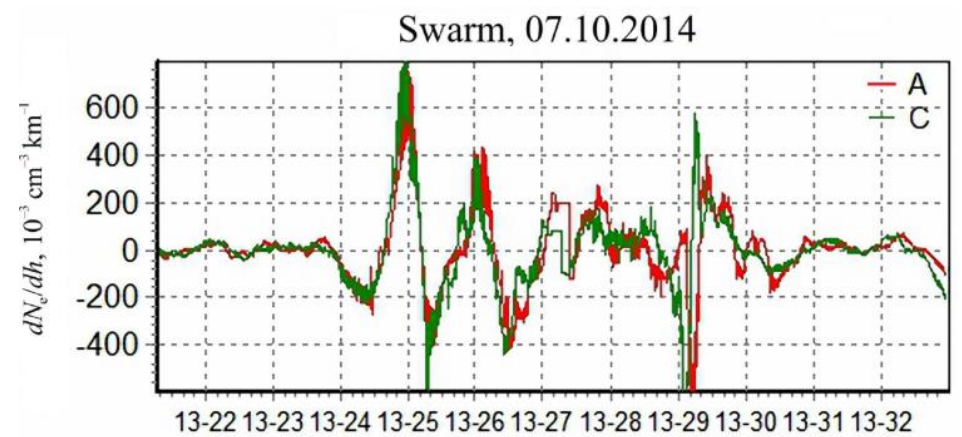

Figure 7. Identification of cyclone effects using the height derivative of electron density for precision tracking by SWARM A and C, on October 7, 2014, 13:20-13:35 UTC

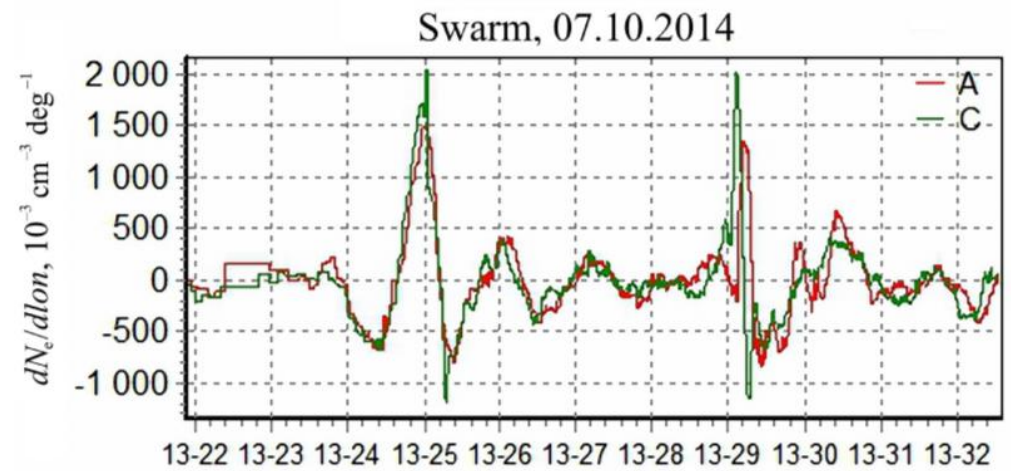

Figure 8. Identification of cyclone effects using the longitude derivative of electron density by SWARM A and C, on October 7, 2014, 13:20-13:35 UTC 
increases in the vicinity of the Brunt-Väisälä frequency [Drobyazko, Krasilnikov, 1985]. For high frequencies, damping sharply increases, therefore at large distances from TC there should be infrasonic waves with frequencies less than $1 \mathrm{mHz}$ and wavelengths of the order of thousands of kilometers. At heights above $100 \mathrm{~km}$, AGWs become nonlinear, thereby giving rise to a complex of dynamic and thermal processes, including the formation of a soliton-like disturbance [Kshevetsky, Gavrilov, 2003].

In the frequency range of the acoustic branch of AGW (<10 min periods) there are also magnetospheric MHD waves observed as Pc5 geomagnetic pulsations. These MHD oscillations are predominantly caused by Alfvén waves. The latter when interacting with the anisotropically conductive lower ionosphere excite the evanescent magnetosonic perturbations that modulate the ionospheric plasma density [Pilipenko et al., 2012]. Meanwhile, Pc5-pulsations are excited at the latitude of the auroral oval, and their amplitude significantly decreases to low latitudes. Therefore, as expected, MHD disturbances cannot make any significant contribution to the observed effects.

In this part of our work, we have tested two techniques for identifying TC effects on the ionosphere using data from LEO satellites. The first is based on the determination of relative $N_{\mathrm{e}}$ variations in the 15 $180 \mathrm{~s}$ frequency band, which correspond to the above spatial scales of AGWs. It is noteworthy that Chou et al. [2017] have also found a concentric response in the ionosphere during passage of strong TC, which is consistent with our findings.

The second technique uses estimates of the spatial electron density derivatives measured by two nearby SWARM satellites. Longitude derivatives, i.e. in the direction perpendicular to the track, prove to be the most informative in terms of identification of the ionospheric response to atmospheric TCs. This is probably due to the fact that when a satellite passes over the disturbance it is the longitude derivative that is directed to the center of the disturbance and hence is maximum.

In conclusion, let us note that the available satellite data in general allow us to identify the ionospheric response to major atmospheric events such as tropical cyclones. Meanwhile, it is currently impossible to unambiguously identify the cause of the electron density modulation in the ionosphere, i.e. to identify the mechanism of this impact (movement of the neutral component, the electric field effect, or plasma transfer due to field-aligned currents), and to interpret our findings we have analyzed the wave modulation mechanism. A slightly different approach is used in the second part of our paper dealing with magnetic disturbances in the TC region.

The SWARM data were provided by the European Space Agency [https://earth.esa.int/web/guest/ swarm/data-access]. This work was supported by RFBR grants Nos. 18-05-00108 (PVA) and 19-0500941 (ZVI), by the State Contracts to MSU (ZVI) and IKI (GVA).

\section{REFERENCES}

Afraimovich E.L., Voyeikov S.V., Ishin A.B., Perevalova N.P., Ruzhin Yu.Ya. Total electron content variations during the powerful typhoon of August 5-11, 2006, near the southeastern coast of China. Geomagnetism and Aeronomy. 2008, vol. 48, no. 5 , pp. 674-679.

Afraimovich E.L., Perevalova N.P. GPS-monitoring verkhnei atmosfery Zemli [GPS monitoring of the Earth's upper atmosphere]. Irkutsk, 2006, 480 p. (In Russian).

Balan N., Souza J., Bailey G.J. Recent developments in the understanding of equatorial ionization anomaly: A review. $J$. Atm. Solar-Terr. Phys. 2018, vol. 171, pp. 3-11.

Bertin E., Testud Y., Kersley L. Medium scale gravity waves in the ionospheric $\mathrm{F}$ region and their possible origin in weather disturbances. Planet. Space Sci. 1975, vol. 23, pp. 493-507.

Bondur V.G., Pulinets S.A., Uzunov D. The effect of largescale atmospheric vortex processes on the ionosphere using hurricane Katrina as an example. Issledovaniya Zemli iz kosmosa [Izvestiya, Atmospheric and Oceanic Physics]. 2008, no. 6, pp. 3-11. (In Russian).

Chou M.Y., Lin C.H., Yue J., Chang L.C., Tsai H.F., Chen C.H. Medium-scale traveling ionospheric disturbances triggered by Super Typhoon Nepartak (2016). Geophys. Res. Lett. 2017, vol. 44, pp. 7569-7577. DOI: 10.1002/2017GL073961.

Danilov A.D., Kazimirovsky E.S., Vergasova G.V., Khachikyan G.Ya. Meteorologicheskie effekty v ionosfere [Meteorological Effects in the Ionosphere]. Leningrad, Gidrometeoizdat Publ., 1987, 267 p. (In Russian).

Drobyazko I.N., Krasil'nikov V.N. Generation of acousticgravity waves by atmospheric turbulence. Radiophysics and Quantum Electronics. 1985, vol. 28, no. 11, pp. 946-952.

Forbes J.M., Palo S.E., Zhang X. Variability of the ionosphere. J. Atm. Solar-Terr. Phys. 2000, vol. 62, pp. 685-693. DOI: 10.1016/S1364-6826(00)00029-8.

Gokhberg M.B., Pilipenko V.A., Pokhotelov O.A., Fedorov E.N. Electromagnetic ELF-noise bursts in the upper atmosphere induced by ground explosions. Geomagnetizm $i$ aeronomiya [Geomagnetism and Aeronomy]. 1996, vol. 36, no. 4, pp. 61-67. (In Russian).

Gossard E.E., Hooke W.H. Waves in the Atmosphere: Atmospheric Infrasound and Gravity Waves, Their Generation and Propagation. Elsevier Scientific Publ. Co., 1975, 456 p.

Ho C.M., Mannucci A.J., Lindqwister U.J., Pi X., Tsurutani B.T. Global ionosphere perturbations monitored by the worldwide GPS network. Geophys. Res. Lett. 1996, vol. 23, iss. 22, pp. 3219-3222. DOI: 10.1029/96GL02763.

Hocke K., Schlegel K. A review of atmospheric gravity waves and traveling ionospheric disturbances: 1982-1995. Ann. Geophys. 1996, vol. 14, iss. 9, pp. 917-940. DOI: 10.1007/ s00585-996-0917-6.

Hofman-Wellenhoft B., Lichtenegger H., Collins J. GPS Theory and Practice. New York, Springer-Verlag Vienna, 1992, $347 \mathrm{p}$.

Huang Y.N., Cheng K., Chen S.W. On the detection of acoustic-gravity waves generated by typhoon by use of real time HF Doppler frequency shift sounding system. Radio Sci. 1985, vol. 20, no. 4, pp. 897-906. DOI: 10.1029/RS020i004p00897.

Isaev N.V., Sorokin V.M., Chmyrev V.M., Serebryakova O.N. Ionospheric electric fields related to sea storms and typhoons. Geomagnetism and Aeronomy. 2002, vol. 42, no. 5, pp. 638-643.

Isaev N.V., Kostin V.M., Belyaev G.G., Ovcharenko O.Ya., Trushkina E.P. Disturbances of the topside ionosphere caused by typhoons. Geomagnetism and Aeronomy. 2010, vol. 50, iss. 2, pp. 243-255. DOI: 10.1134/S001679321002012X. 
Kshevetsky S.P., Gavrilov N.M. Vertical propagation and breaking of nonlinear gravity waves in the atmosphere. Geomagnetism and Aeronomy. 2003, vol. 43, no. 1, p. 69.

Li W., Yue J., Wu S., Yang Y., Li Z., Bi J., Zhang K. Ionospheric responses to typhoons in Australia during 2005-2014 using GNSS and FORMOSAT-3/COSMIC measurements. GPS Solutions. 2018, vol. 22, no. 3, pp. 1-22. DOI: 10.1007/s10291018-0722-1.

Melioransky A.S. Vysypanie elektronov iz radiatsionnykh poyasov $i$ koltsevogo toka pod vliyaniem izluchenii taifunov $v$ severo-zapadnoi chasti Tikhogo okeana. Taifun "Maik" $i$ moshchnyi tropicheskii shtorm "Nell" [Electron precipitation from radiation belts and ring current under the effect of typhoon emissions in the north-western part of the Pacific Ocean. Typhoon Mike and powerful tropical storm Nell]. Preprint of Space Research Institute. No.2136. Moscow, 2007, 18 p.

Olsen N., Friis-Christensen E., Floberghagen R., Alken P., Beggan C.D., Chulliat A., et al. The Swarm Satellite Constellation Application and Research Facility (SCARF) and Swarm data products. Earth, Planets and Space. 2013, vol. 65 , pp. 1189-1200. DOI: 10.5047/eps.2013.07.001.

Pilipenko V., Belakhovsky V., Kozlovsky A., Fedorov E., Kauristie K. Determination of the wave mode contribution into the ULF pulsations from combined radar and magnetometer data: Method of apparent impedance. J. Atm. Solar-Terr. Phys. 2012, vol. 77, pp. 85-95. DOI: 10.1016/j.jastp.2011.11.013.

Pokhotelov O.A., Pilipenko V.A., Fedorov E.N., Stenflo L., Shukla P.K. Induced electromagnetic turbulence in the ionosphere and the magnetosphere. Physica Scripta. 1994, vol. 50, pp. 600-605.

Polyakova A.S., Perevalova N.P. Comparative analysis of TEC disturbances over tropical cyclone zones in the North-West Pacific Ocean. Adv. Space Res. 2013, vol. 52, pp. 1416-1426. DOI: 10.1016/j.asr.2013.07.029.

Rishbeth H. F-region links with the low atmosphere? $J$. Atmos. Solar-Terr. Phys. 2006, vol. 68, no. 3, pp. 469-478. DOI: 10.1016/j.jastp.2005.03.017.

Rozhnoi A., Solovieva M., Levin B., Hayakawa M., Fedun vol. Meteorological effects in the lower ionosphere as based on VLF/LF signal observations. Natural Hazards Earth System Sci. 2014, vol. 14, pp. 2671-2679.
Sorokin V.M., Isaev N.V., Yaschenko A.K., Chmyrev V.M., Hayakawa M. Strong DC electric field formation in the low latitude ionosphere over typhoons. J. Atm. Solar-Terr. Phys. 2005, vol. 67, pp. 1269-1279. DOI: 10.1016/j.jastp.2005.06.014.

Vanina-Dart L.B., Pokrovskaya I.V., Sharkov E.A. Studying the interaction between the lower equatorial ionosphere and tropical cyclones according to data of remote and rocket sounding. Izvestiya, Atmospheric and Oceanic Physics. 2007, no. 2, pp. 19-27.

Walker G.O. Longitudinal structure of the F-region equatorial anomaly - a review. J. Atmos. Terr. Phys. 1981, vol. 43, iss. 8, pp. 763-774. DOI: 10.1016/0021-9169(81)90052-0.

Xiao Z., Xiao S.G., Hao Y.Q., Zhang D.H. Morphological features of ionospheric response to typhoon. J. Geophys. Res. 2007, vol. 112, iss. A4, A04304. DOI: 10.1029/2006JA011671.

Zakharov V.I., Kunitsyn V.E. Regional features of atmospheric manifestations of tropical cyclones according to groundbased GPS network data. Geomagnetism and Aeronomy. 2012, vol. 52, no. 4, pp. 533-545. DOI: 10.1134/S0016793212040160.

Zakharov V.I., Zienko A.S., Kunitsyn V.E. GPS signal propagation under varied solar activity. Elekromagnitnye volny $i$ elektronnye sistemy [J. Electromagnetic Waves and Electronic Systems]. 2008, vol. 13, no. 8, pp. 51-57. (In Russian).

URL: http://directory.eoportal.org/web/eoportal/satellitemissions/s/swarm (accessed March 1, 2019).

URL: http://weather.unisys.com/hurricane (accessed March 1, 2019).

URL: http://www.nhc.noaa.gov/aboutsshws.php (accessed March 1, 2019).

URL: http://directory. eoportal.org/web/eoportal/satellitemissions/s/swarm (accessed March 1, 2019).

URL: https://earth.esa.int/web/guest/ swarm/data-access (accessed March 1, 2019).

How to cite this article

Zakharov V.I., Pilipenko V.A., Grushin V.A., Khamidullin A.F. Impact of Typhoon Vongfong 2014 on the ionosphere and geomagnetic field according to SWARM satellite data: 1. Wave disturbances of ionospheric plasma. Solar-Terrestrial Physics. 2019. Vol. 5. Iss. 2. P. 101-108. DOI: $10.12737 /$ stp-52201914. 